\title{
Efficacy and Safety of Transanal Tube Drainage for Prevention of Anastomotic Leakage Following Laparoscopic Low Anterior Resection for Rectal Cancers
}

\author{
Yasutake Uchima*, Naoki Aomatsu, Hironari Miyamoto, Takuma Okada, Shigeaki Kurihara, \\ Toshiki Hirakawa, Takehiko Iwauchi, Junya Morimoto, Shigehito Yamagata, \\ Kazunori Nakazawa, Kazuhiro Takeuchi
}

Department of Surgery, Fuchu Hospital, Izumi city, Osaka, Japan

Email: *y_uchima@seichokai.or.jp

How to cite this paper: Uchima, Y., Aomatsu, N., Miyamoto, H., Okada, T., Kurihara, S., Hirakawa, T., Iwauchi, T., Morimoto, J., Yamagata, S., Nakazawa, K. and Takeuchi, K. (2018) Efficacy and Safety of Transanal Tube Drainage for Prevention of Anastomotic Leakage Following Laparoscopic Low Anterior Resection for Rectal Cancers. Journal of Cancer Therapy, 9, 538-544.

https://doi.org/10.4236/jct.2018.97045

Received: May 22, 2018

Accepted: July 7, 2018

Published: July 10, 2018

Copyright (c) 2018 by authors and Scientific Research Publishing Inc. This work is licensed under the Creative Commons Attribution International License (CC BY 4.0).

http://creativecommons.org/licenses/by/4.0/

(c) (i) Open Access

\begin{abstract}
Introduction: Laparoscopic surgery is widely used for the treatment of colorectal cancer. But anastomotic leakage is one of the most serious complications following laparoscopic low anterior resection (LAR) for rectal cancer. The purpose of this study was to investigate whether transanal drainage tube placement can reduce anastomotic leakage and avoid re-operation after laparoscopic LAR. Methods: Retrospective assessment was performed on 143 patients with rectal cancers who underwent laparoscopic LAR between April 2009 and March 2016. A diverting stoma was not created in all 143 patients. A transanal drainage tube was placed after anastomosis using a double stapling technique, in 90 patients (group TT). In group TT, a $24 \mathrm{Fr}$. Silicon catheter was inserted into the anus and was placed approximately $20-25 \mathrm{~cm}$ in the descending colon. Another 53 patients were operated on without a transanal drainage tube (group NTT). Clinicopathological and operative variables, the frequencies of anastomotic leakage and re-operation after leakage were investigated. Results: Between the two groups (Group TT and NTT), age, gender, body mass index, tumor size, Dukes' stage, the number of Liniar stapler firings for rectal transaction, and the rate of left colic artery preservation were comparable. Intra-operative blood loss and operation time decreased group TT from group NTT $(\mathrm{p}<0.05)$. The frequency of leakage was $2.8 \%(2 / 69)$ in group TT and was $13.2 \%(7 / 53)$ in group NTT. The rate of leakage was significantly lower in group TT $(\mathrm{p}=0.03)$. Furthermore, the re-operation rate for symptomatic anastomotic leakage was $0 \%(0 / 2)$ in group $\mathrm{TT}$, while in contrast it was $28.5 \%(2 / 7)$ in group NTT. The rate of re-operation was lower in group TT than group NTT $(\mathrm{p}=0.07)$ and all cases with symptomatic leakage in
\end{abstract}


group TT were cured by conservative treatment. There was no side effect of transanal drainage tube (perforation or bleeding of the colon, or deviation of the tube) in Group TT. Conclusions: Transanal drainage tube placement prevents anastomotic leakage after laparoscopic LAR. We regard the transnanal drainage tube to be safe and effective without side effect.

\section{Keywords}

Rectal Cancer, Anastomosis Leakage, Transanal Drainage Tube

\section{Introduction}

Anastomotic leakage is one of the most severe complications related to colorectal cancer surgery. It contributes not only to postoperative morbidity and mortality, but also to local recurrence and poor prognosis [1] [2] [3]. Anastomotic leakage may prolong hospital stay and have deleterious effects on oncologic outcomes and postoperative quality of life [4]. Therefore, prevention of anastomotic leakage is crucial or safe rectal anastomosis. Intraluminal pressure in the anastomotic portion may be associated with anastomotic leakage. Transanal tube placement may reduce postoperative anastomotic leakage rate by reducing intraluminal pressure and preventing fecal extrusion through the staple line [5]. The purpose of this study was to investigate whether transanal tube placement can reduce anastomotic leakage following laparoscopic LAR.

\section{Materials and Methods}

\subsection{Patients}

Between April, 2009 and March, 2016, 143 patients underwent rectal cancer surgery with double stapling technique (DST) reconstruction in our institution. The inclusion criteria for this retrospective study were a primary tumor below the rectum, and curative resection accomplished by anterior resection with DST reconstruction. Rectal cancer was diagnosed histologically by biopsy under colonoscopy. Patients with stoma placement and patients undergoing additional surgical procedures concomitantly were excluded from the analysis. A diverting stoma was not created in all 143 patients. A transanal tube was placed after anastomosis using a double stapling technique, in 90 patients (group TT) between October, 2012 and March, 2016. In group TT, a 24 Fr. Silicon catheter (Phycon tube, Fuji Systems Corporation) was inserted into the anus (Figure 1(a) and Figure 1(b)). The top of the group TT was placed approximately $20-25 \mathrm{~cm}$ in the descending colon (Figure 2(d)). Another 53 patients were operated on without a transanal tube (group NTT), between April, 2009 and September. 2012. We began use of the transanal tube in October 2012, thus all of the cases in goup TT were rectal cancer surgeries perfomed between October, 2012 and March, 2016. Clinicopathological and operative variables, the frequencies of 
anastomotic leakage and re-operation after leakage were investigated.

\subsection{Surgical Technique}

Operations were performed laparoscopic surgery. Laparoscopic surgery was performed using a five-port technique. We dissected and mobilized the sigmoid colon via the medial-to-lateral approach in Toldt's space, then performed a radical proximal lymph node dissection, following the Japanese Society for Cancer of the Colon and Rectum (JSCCR) guidelines. The length of bowel transected was determined by the JSCCR guidelines, in which more than a 2 -cm distal margin in middle and lower rectal cancer. For middle or lower rectal cancers, the rectum was dissected posteriorly to the level of the levator muscle and a total mesorectal excision (TME) was carried out.

Before transection, the bowel was clamped at the anal side of the tumor and washed out through the anus with $500 \mathrm{ml}$ of the normal saline. The bowel was transected using Linear staplers (Figure 2(a)), and the proximal end of the bowel was delivered through a small incision. The bowel was then resected extracorporeally and an anvil was placed in the proximal colon. An anastomosis was performed with DST under laparoscopy (Figure 2(b)) and an abdominal drain (Clio-drain, Sumitomo Bakelite Co., Ltd.) was placed near the anastomosis (Figure 2(c)), in all patients.

\subsection{Postoperative Course}

Oral intake was resumed between postoperative days (PODs) 3 and 4 and most transanal tubes were removed on PODs 4 - 5 .

\subsection{Definition of Anastomotic Leakage}

Aanastomotic leakage in this study was defined as either purulent discharge from the abdominal drain or a peri-anastomotic abscess detected by CT. In the case of turbid discharge with high fever, we comprehensively diagnosed anastomotic, based on CT findings.

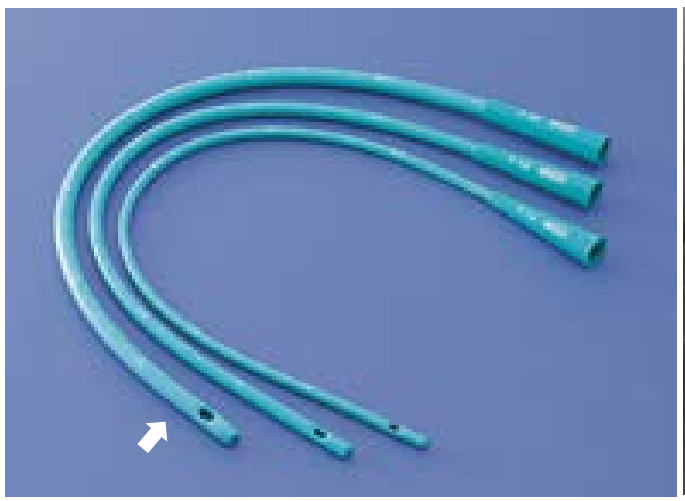

(a)

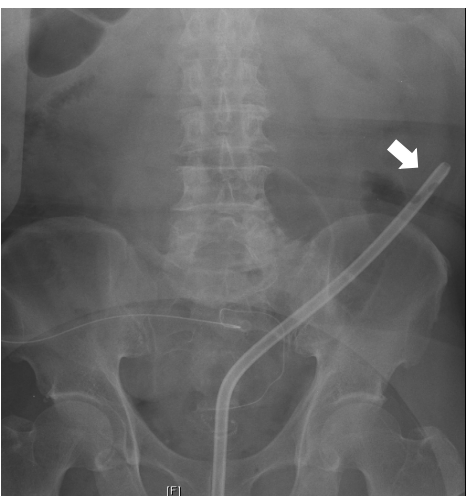

(b)

Figure 1. (a) 24 Fr. Silicon catheter was inserted into the anus. (b) Postoperative X-ray. The top of the group TT was placed approximately $20-25 \mathrm{~cm}$ in the descending colon. 


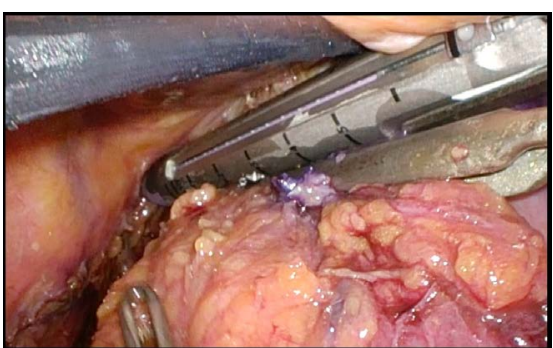

(a)

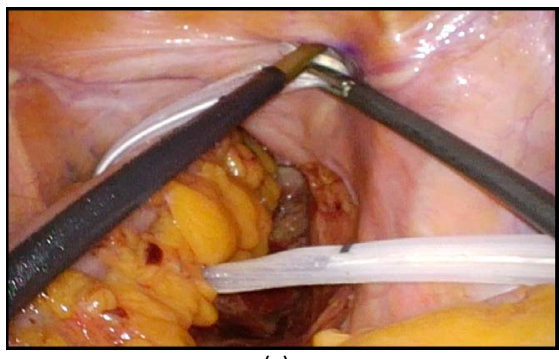

(c)

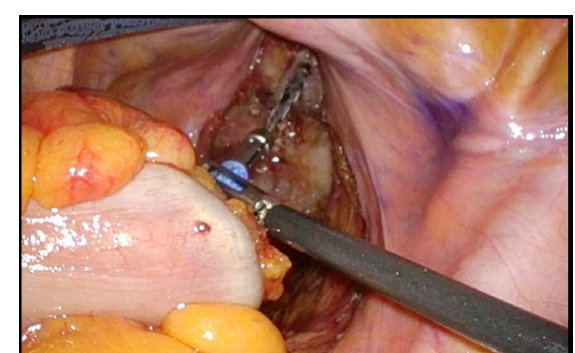

(b)

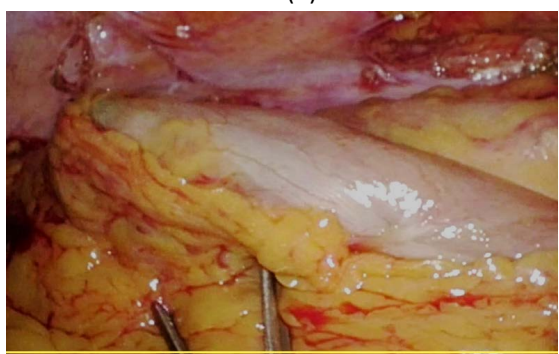

(d)

Figure 2. (a) The bowel was transected using Linear staplers. (b) An anastomosis was performed with DST under laparoscopy. (c) An abdominal drain was placed near the anastomosis. (d) The top of the group TT was placed approximately $20-25 \mathrm{~cm}$ in the descending colon.

\subsection{Ethical Considerations}

This study was performed in accordance with the Declaration of Helsinki and institutional guidelines. Written informed consent was obtained from all patients, between October, 2012 and March, 2016, who underwent rectal surgery and transanal tube placement. This study was approved by the Ethical Committee of the Fuchu Hospital 2012.

\subsection{Statistical Analysis}

Statistical analyses were performed using by the Statcel 4 software program (OMS, Saitama, Japan). The collected patient data were reviewed. X2 test and student's $t$ test for categorical variables were used for statistical comparison of characteristics and complications of patients in both groups. All $\mathrm{p}$ values were two-sided, and a $\mathrm{p}$ value $<0.05$ was considered significant.

\section{Results}

Between April, 2009 and March, 2016, 143 patients underwent rectal cancer surgery with DST reconstruction in our institution. A transanal tube was placed after anastomosis using a DST, in 90 patients (group TT). Another 53 patients were operated on without a transanal tube (group NTT).

Patient age, gender, body mass index, tumor size, Dukes' stage, the number of liniar stapler firings for rectal transaction, and the rate of left colic artery preservation were comparable between the two groups.

Intra-operative blood loss and operation time decreased group TT from group NTT (blood loss: $\mathrm{p}=0.04$, operation time: $\mathrm{p}=0.06$ ). 
The frequency of leakage was $2.2 \%$ (2/90) in group TT and was $13.2 \%(7 / 53)$ in group NTT. The rate of leakage was significantly lower in group TT ( $\mathrm{p}=$ 0.04).

Furthermore, the re-operation rate for symptomatic anastomotic leakage was $0 \%(0 / 2)$ in group TT, while in contrast it was $28.5 \%(2 / 7)$ in group NTT. The rate of re-operation was lower in group TT than group NTT $(\mathrm{p}=0.06)$ and all cases with symptomatic leakage in group TT were cured by conservative treatment (Table 1). There was no side effect of transanal drainage tube placement (perforation or bleeding of the colon, or deviation of the tube).

\section{Discussion}

Anastomosis leakage is a common and potentially serious postoperative complication of colorectal cancer surgery. It is associated with increased mortality and morbidity and may require a prolonged postoperative hospital stay or a second surgical procedure, such as for a diverting stoma. Anastomosis leakage causes increased physical, emotional, and financial stress, and it may increase the risk of local recurrence and thereby negatively impact survival [6].

It has been reported that male gender, low tumor location, low anastomosis, preoperative chemoradiation, and advanced cancer stage increased the risk of anastomotic leakage following LAR for rectal cancer [7] [8] [9] [10]. LCA preservation is important to reduce anastomotic leakage [11]. Furthermore, endoluminal pressure in the anastomotic portion is resumed to be associated with anastomotic leakage [12]. With the development of surgical instruments and surgical techniques, sphincter-preserving procedures have become more prevalent [13]. However, the risk of anastomotic leakage is increasing, and placement of a transanal tube for its prevention is controversial [14] [15].

Table 1. Clinical and surgical factors. Rate of anastonotic leakage and re-operation.

\begin{tabular}{|c|c|c|c|}
\hline & NTT Group n $=53$ & TT Group $n=90$ & $\mathrm{p}$ value \\
\hline Age, year & $66.9 \pm 10.2$ & $67.3 \pm 8.4$ & 0.82 \\
\hline Gender (male/female) & $34 / 19$ & $39 / 24$ & 0.6 \\
\hline Body mass index, $\mathrm{kg} / \mathrm{m}^{2}$ & $22.2 \pm 2.7$ & $22.3 \pm 3.5$ & 0.89 \\
\hline Tumor size $(\mathrm{cm})$ & $41.4 \pm 18.8$ & $35.7 \pm 17.3$ & 0.09 \\
\hline Dukes' stage A/B/C & $20 / 16 / 17$ & $32 / 28 / 30$ & 0.76 \\
\hline Operation time (min) & $183.1 \pm 43.4$ & $166.4 \pm 48.7$ & 0.06 \\
\hline Blood loss (ml) & $56.6 \pm 88.2$ & $28.2 \pm 57.6$ & 0.04 \\
\hline Preserving Left colic Artery & $49(92.5 \%)$ & $41(45.6 \%)$ & 0.05 \\
\hline Number of staple firing & 1.18 & 1.15 & 0.63 \\
\hline Anastomotic leakage & $7(13.2 \%)$ & $2(2.2 \%)$ & 0.03 \\
\hline Re-operation & $2(3.7 \%)$ & $0(0 \%)$ & 0.07 \\
\hline Postoperative stay, day & $14.7 \pm 22.3$ & $9.9 \pm 5.1$ & 0.11 \\
\hline
\end{tabular}


The meta-analysis was conducted to evaluate existing data to help clarify the role of transanal tube placement in the prevention of anastomotic leakage and reoperation. Results of both the meta-analysis of the three observational studies and the one randomized trials indicate that placement of a transanal tube significantly decreases the risk of leakage and reoperation [16]. While the mechanism of action of the transanal tube is unclear, it may be related to reduction in endoluminal pressure in the anastomotic portion of the intestine which may be an important factor in anastomotic leakage [17]. Although transanal tube may reduce the risk of anastomotic leakage and reoperation, its placement increases patients' discomfort and inconvenience. There have also been reports of tubes perforating bowel especially in the region anterior to the sacrum [18]. However, compared with the reoperations required for anastomotic leakage in the absence of a transanal tube, complications associated with a transanal tube are relatively minor.

Our study demonstrates the effectiveness of TT placement for the prevention of anastomosis leakage after rectal cancer surgery with DST reconstruction. The frequency of leakage was $2.2 \%(2 / 90)$ in group TT and was $13.2 \%(7 / 53)$ in group NTT. The rate of leakage was significantly lower in group TT $(\mathrm{p}<0.05)$.

Furthermore, the re-operation rate for symptomatic anastomotic leakage was $0 \%(0 / 2)$ in group TT, while in contrast it was $28.5 \%(2 / 7)$ in group NTT. The rate of re-operation was lower in group TT than group NTT $(\mathrm{p}<0.05)$ and all cases with symptomatic leakage in group TT were cured by conservative treatment.

In conclusion, our study shows that TT placement may prevent anastomotic leakage after rectal cancer surgery with DST reconstruction. TT placement was effective for prevention of anastomotic leakage following laparoscopic LAR and avoiding re-operation after symptomatic leakage. We regard the transanal drainage tube to be safe and effective without side effect.

\section{References}

[1] Branagan, G. and Finnis, D. (2005) Prognosis after Anastomotic Leakage in Colorectal Surgery. Diseases of the Colon \& Rectum, 48, 1021-1026.

https://doi.org/10.1007/s10350-004-0869-4

[2] Den Dulk, M., Marijinen, C.A.M., Collette, L., et al. (2009) Multicentre Analysis of Oncological and Survival Outcomes Following Anastomotic Leakage after Rectal Cancer Surgery. British Journal of Surgery, 96, 1066-1075. https://doi.org/10.1002/bjs.6694

[3] Mrnezami, A., Mirnezami, R., Chandrakumaran, K., et al. (2011) Increased Local Recurrence and Reduced Survival from Colorectal Cancer Following Anastomotic Leak: Systematic Review and Meta-Analysis. Annals of Surgery, 253, 890-899. https://doi.org/10.1097/SLA.0b013e3182128929

[4] Wang, J. (2005) Principle and Complications of Aus-Preserving Operation for Low Rectal Cancer. Journal of Clinical Surgery, 13, 678-679.

[5] Ha, G.W., Kim, H.J. and Lee, M.R. (2015) Transanal Tube Placement for Prevention of Anastomotic Leakage Following Low Anterior Resection for Rectal Cancer: A Systematic Review and Meta-Analysis. Annals of Surgical Treatment and Research, 
89, 313-318.

[6] Matsuda, M., Turuta, M., Hasegawa, H., et al. (2016) Transanal Drainage Tube Placement to Prevent Anastomotic Leakage Following Colorectal Cancer Surgery with Double Stapling Reconstruction. Surgery Today, 46, 613-620.

https://doi.org/10.1007/s00595-015-1230-3

[7] Vignali, A., Fazio, V.W., Lavery, I.C., Milsom, J.W., et al. (1997) Factors Associated with the Occurrence of Leaks in Stapled Rectal Anastomoses: A Review of $1014 \mathrm{~Pa}$ tients. Journal of the American College of Surgeons, 185, 105-113.

https://doi.org/10.1016/S1072-7515(97)00018-5

[8] Yamamoto, S., Fujita, S., Akasu, T., et al. (2012) Risk Factors for Anastomotic Leakage after Laparoscopic Surgery for Rectal Cancer Using a Stapling Technique. Surgical Laparoscopy Endoscopy \& Percutaneous Techniques, 22, 239-243.

[9] Lee, W.S., Yun, S.H., Roh, Y.N., et al. (2008) Risk Factors and Clinical Outcome for Anastomotic Leakage after Total Mesorectal Excision for Rectal Cancer. World Journal of Surgery, 32, 1124-1129. https://doi.org/10.1007/s00268-007-9451-2

[10] Park, J.S., Choi, G.S., Kim, S.H., et al. (2013) Multicenter Analysis of Risk Factors for Anastomotic Leakage after Laparoscopic Rectal Cancer Excision: The Korean Laparoscopic Colorectal Surgery Study Group. Annals of Surgery, 257, 665-671. https://doi.org/10.1097/SLA.0b013e31827b8ed9

[11] Hinoi, T., Okajima, M., Shimomura, M., et al. (2013) Effect of Left Colic Artery Preservation on Anastomotic Leakage in Laparoscopic Anterior Resection for Middle and Low Rectal Cancer. World Journal of Surgery, 37, 2935-2943.

https://doi.org/10.1007/s00268-013-2194-3

[12] Guenaga, K.F., Lustosa, S.A., Saad, S.S., et al. (2008) Ileostomy or Colostomy or Temporary Decompression of Colorectal Anastomosis. Systematic Review and Meta-Analyssis. Acta Cirúrgica Brasileira, 23, 294-303. https://doi.org/10.1590/S0102-86502008000300014

[13] Bordeianou, L., Maguire, L.H., Alavi, K., Sudan, R., Wise, P.E. and Kaiser, A.M. (2014) Sphincter-Sparing Surgery in Patients with Low-Lying Rectal Cancer: Techniques, Oncologic Outcomes, and Functional Results. Journal of Gastrointestinal Surgery, 18, 1358-1372. https://doi.org/10.1007/s11605-014-2528-y

[14] Moran, B.J. (2010) Predicting the Risk and Diminishing the Consequences of Anastomotic Leakage after Anterior Resection for Rectal Cancer. Acta Chirurgica Iugoslavica, 57, 47-50. https://doi.org/10.2298/ACI1003047M

[15] Yang, L., Huang, X.E. and Zhou, J.N. (2013) Risk Assessment on Anastomotic Leakage after Rectal Cancer Surgery: An Analysis of 753 Patients. Asian Pacific Journal of Cancer Prevention, 14, 4447-4453. https://doi.org/10.7314/APJCP.2013.14.7.4447

[16] Guenaga, K.F., Lustosa, S.A., Saad, S.S., Saconato, H. and Matos, D. (2007) Ileostomy or Colostomy for Temporary Decompression of Colorectal Anastomosis. The Cochrane Database of Systematic Reviews, 1, CD004647. https://doi.org/10.1002/14651858.CD004647.pub2

[17] Wang, S., Zhang, Z., Liu, M., et al. (2016) Efiicacy of Transanal Tube Placement after Anterior Resection for Rectal Cancer: A Systematic Review and Meta-Analysis. World Journal of Surgical Oncology, 14, 92. https://doi.org/10.1186/s12957-016-0854-0

[18] Nishigori, H., Ito, M., Nishizawa, Y., Nishizawa, Y., Kobayashi, A., Sugito, M., et al. (2014) Effectiveness of a Transanal Tube for the Prevention of Anastomotic Leakage after Rectal Cancer Surgery. World Journal of Surgery, 38, 1843-1851.

https://doi.org/10.1007/s00268-013-2428-4 